\title{
Adenovirus $F$ protein as a delivery vehicle for botulinum B
}

\author{
Beata Clapp', Sarah Golden', Massimo Maddaloni' ${ }^{1}$ Herman F Staats² and David W Pascual*1
}

\begin{abstract}
Background: Immunization with recombinant carboxyl-terminal domain of the heavy chain (Hc domain) of botulinum neurotoxin (BoNT) stimulates protective immunity against native BoNT challenge. Most studies developing a botulism vaccine have focused on the whole $\mathrm{Hc}$; however, since the principal protective epitopes are located within $\beta$-trefoil domain ( $\mathrm{Hc} \beta$ tre), we hypothesize that immunization with the $\mathrm{Hc} \beta$ tre domain is sufficient to confer protective immunity. In addition, enhancing its uptake subsequent to nasal delivery prompted development of an alternative vaccine strategy, and we hypothesize that the addition of targeting moiety adenovirus 2 fiber protein (Ad2F) may enhance such uptake during vaccination.
\end{abstract}

Results: The Hcßtre serotype B immunogen was genetically fused to $\mathrm{Ad} 2 \mathrm{~F}$ ( $\mathrm{Hc} \beta \mathrm{Btre} / \mathrm{B}-\mathrm{Ad} 2 \mathrm{~F}$ ), and its immunogenicity was tested in mice. In combination with the mucosal adjuvant, cholera toxin (CT), enhanced mucosal IgA and serum IgG Ab titers were induced by nasal $\mathrm{Hc} \beta$ tre-Ad2F relative to $\mathrm{Hc} \beta$ tre alone; however, similar Ab titers were obtained upon intramuscular immunization. These BoNT/B-specific Abs induced by nasal immunization were generally supported in large part by Th2 cells, as opposed to Hcßtre-immunized mice that showed more mixed Th1 and Th2 cells. Using a mouse neutralization assay, sera from animals immunized with $\mathrm{Hc} \beta$ tre and $\mathrm{Hc} \beta$ tre-Ad2F protected mice against $2.0 \mathrm{LD}_{50}$.

Conclusion: These results demonstrate that $\mathrm{Hc} \beta$ tre-based immunogens are highly immunogenic, especially when genetically fused to Ad2F, and Ad2F can be exploited as a vaccine delivery platform to the mucosa.

\section{Background}

Clostridium botulinum is a Gram-positive, spore-forming anaerobe commonly found in soil, and is responsible for production of botulinum neurotoxin (BoNT), a potent lethal toxin [1]. Seven BoNT serotypes (A-G) have been identified [2]: serotypes $A, B, E$, and $F$ are primarily responsible for human botulism; serotypes $\mathrm{C}$ and $\mathrm{D}$ are mostly limited to intoxication of animals [3]; and host specificity for serotype $G$ has not been fully determined [4]. Three clinical manifestations are related to naturally acquired botulism: foodborne, infant, and wound botulism [5]. However, recently, three other forms have been described [6]: undefined or adult intestinal, inadvertent injection-related and inhalational botulism. All forms of BoNT intoxication present with the same clinical syndrome of symmetrical and descending flaccid paralysis of

* Correspondence: dpascual@montana.edu

1 Veterinary Molecular Biology, Montana State University, Bozeman, MT $59717-$ 3610, USA

Full list of author information is available at the end of the article motor and autonomic nerves, which can cause respiratory arrest and death $[7,8]$. Since natural exposure to $C$. botulinum spores is rare, the necessity to create a vaccine is primarily for high-risk individuals. Moreover, an intentional release to contaminate food and water supplies or for use as an aerosolized weapon [3] warrants the development of vaccines to protect high-risk groups.

Currently, there are no certified vaccines for preventing human botulism. In the United States, an investigational pentavalent botulinum toxoid that includes serotypes A through $\mathrm{E}$ is used to vaccinate high risk individuals [9]. Although toxoid-based vaccines can be conveniently produced, these have a number of limitations, including isolation of active toxins, loss of essential neutralizing epitopes by formalin treatment, and multiple immunizations required to sustain elevated Ab titers [10]. To overcome these limitations, recent efforts have shifted to adapting recombinant BoNT heavy $(\mathrm{H})$ chain to develop subunit vaccines $[11,12]$, as well as employing DNA vac- 
cine approaches $[13,14]$. Such approaches eliminate the reliance on handling active intact BoNTs.

BoNTs are originally produced as $150 \mathrm{kDa}$ chains that are post-translationally nicked into a $\mathrm{C}$-terminal $\mathrm{H}$ chain (ca. $100 \mathrm{kDa}$ ) and $\mathrm{N}$-terminal light (L) chain (ca. $50 \mathrm{kDa})$ [13]. Functionally, the toxins contain three domains [15]: a receptor-binding domain, a translocation domain, and an enzymatic domain. Part of the receptor-binding domain is located in the carboxyl-terminal segment of the $\mathrm{H}$ chain, a region referred as the fragment $\mathrm{C}$ or $\mathrm{Hc}$. The amino-terminal segment of the $\mathrm{H}$ chain is responsible for the translocation and internalization of the $\mathrm{L}$ chain into the cell [16]. The $\mathrm{L}$ chain acts as a zinc-dependent endoprotease, which selectively attacks three crucial proteins, disabling the docking and fusion of acetylcholinecontaining synaptic vesicles to the plasma membrane [17].

A number of studies [rev. in [9]] have reported that recombinant $50 \mathrm{kDa} \mathrm{Hc}$ domain possesses the ability to stimulate protective Abs capable of neutralizing native BoNT. The Hc is an effective immunogen and simultaneously capable of binding and penetrating epithelial barriers in the gut and airway, suggesting that this molecule could be used as a mucosal vaccine against botulism [18]. The crystal structure of BoNT shows that the Hc fragment is composed of two subdomains: N-terminal developing jelly roll motif and a $\mathrm{C}$-terminal forming $\beta$-trefoil domain $[19,20]$. The $\beta$-trefoil motif, especially the final approximately 150 residues, significantly diverges among serotypes with sequence similarity as low as $15 \%$ [21], but maintains a ganglioside binding motif that is conserved [22]. Since there is a lack of significant cross-reactivity among the serotypes, it is speculated that the protective epitopes are located within this fragment of Hc. In our previous study, vaccination with the Hc $\beta$-trefoil domain, referred to as $\mathrm{Hc} \beta$ tre/A, rather than the intact $\mathrm{Hc}$, was shown to protect against BoNT/A [23].

A major impediment in forwarding mucosal vaccines into humans is the absence of mucosal adjuvants suitable for human use. Significant efforts have focused on live, attenuated vaccine approaches to circumvent the requirement for exogenous adjuvants. One such vaccine vector has been a modified adenovirus widely tested for a number of experimental vaccines [24]. A major benefit of adenoviral vectors is their affinity for the mucosal epithelium, which makes them optimal for mucosal vaccination [25], although this may be problematic for use in humans because of pre-existing Abs [26]. Initial attachment of adenovirus to the host cell surface via coxsackieadenovirus receptor is mediated by the knob domain of adenovirus fiber [27]. To circumvent use of a live vector, the adenovirus 2 fiber (Ad2F) protein was selected and tested for its abilities to deliver BoNT/B Hc $\beta$ tre vaccine. Previously, immunization with the fusion protein,
Hc $\beta$ tre/A-Ad2F, increased onset of plasma and mucosal $\mathrm{Ab}$ responses and conferred protection against BoNT/A [23]. In light of these findings, we queried whether this approach could be adapted to generate a vaccine for $\mathrm{BoNT} / \mathrm{B}$. Thus, $\mathrm{Hc} \beta$ tre/B was genetically fused to Ad2F, and immunization with Hc $\beta$ tre/B-Ad2F was found to be more immunogenic than $\mathrm{Hc} \beta$ tre alone, further suggesting the utility of Ad2F as a targeting moiety to improve a mucosal vaccine's immunogenicity.

\section{Results}

Comparison of $\mathrm{Ab}$ responses by $\mathrm{Hc} \beta$ tre-or $\mathrm{Hc} \beta$ tre-Ad2Fvaccinated mice

Groups of mice were immunized by the intranasal (i.n.) route with either $\mathrm{Hc} \beta$ tre or $\mathrm{Hc} \beta$ tre-Ad2F to assess the ability of each to evoke a mucosal and systemic immune response (Figure 1A,B). In parallel experiments, mice were given the same vaccines, but via the i.m. route (Figure $1 \mathrm{C}, \mathrm{D})$. All mice were boosted on days 7 and 14 . Serum and fecal samples were collected at weekly intervals beginning on day 14 until day 35 post-primary immunization and then evaluated for anti-Hcßtre $A b$ titers by standard ELISA methods. Significant S-IgA Ab responses were detected in fecal extracts from mice dosed nasally with Hc $\beta$ tre-Ad2F, either in the presence or absence of CT adjuvant, as opposed to mice dosed nasally with $\mathrm{Hc} \beta$ tre, which produced weak $\mathrm{Hc} \beta$ tre-specific S-IgA Abs (Figure1A). The intramuscular (i.m.) Hc $\beta$ tre-Ad2Fimmunized mice also exhibited elevated fecal $\mathrm{Hc} \beta$ trespecific S-IgA Ab titers, but these tended to wane at the later time points (Figure $1 \mathrm{C}$ ). In the presence of an adjuvant, i.n. (Figure 1B) and i.m. immunization (Figure 1D) with $\mathrm{Hc} \beta$ tre-Ad2F and $\mathrm{Hc} \beta$ tre induced elevated serum IgG Abs. Although mice i.n. immunized with $\mathrm{Hc} \beta$ treAd2F alone or i.m. or i.n. with $\mathrm{Hc} \beta$ tre alone exhibited only weak serum IgG responses (Figure 1B,D), mice i.m. immunized with $\mathrm{Hc} \beta$ tre-Ad2F without CT showed modest induction of serum IgG Ab responses (Figure 1D).

To assess whether S-IgA anti-Hc $\beta$ tre Abs were induced in nasal secretions, nasal wash samples collected on day 35 post-primary immunization showed significantly elevated S-IgA Abs in those mice immunized i.n. with $\mathrm{Hc} \beta$ tre-Ad2F plus $\mathrm{CT}$ when compared with mice given adjuvanted Hc $\beta$ tre (Figure 2A). Mice dosed with the vaccines, but without adjuvant, showed no S-IgA Abs in their nasal washes (Figure 2A). Sera from the immunized mice were also evaluated for the induced IgG subclass responses from the $\mathrm{CT}$ co-immunized mice (Figure $2 B, C)$. Both groups of i.n.-immunized mice showed elevations in their IgG1, IgG2a, and IgG2b anti-Hc $\beta$ tre responses, but only the Hc $\beta$ tre-Ad2F plus CT-immunized mice showed significant elevations in IgG1 responses ( $\mathrm{p} \leq 0.001)$ (Figure $2 \mathrm{~B})$. In contrast, the i.m. Hc $\beta$ tre-Ad2F plus CT-immunized mice showed signifi- 


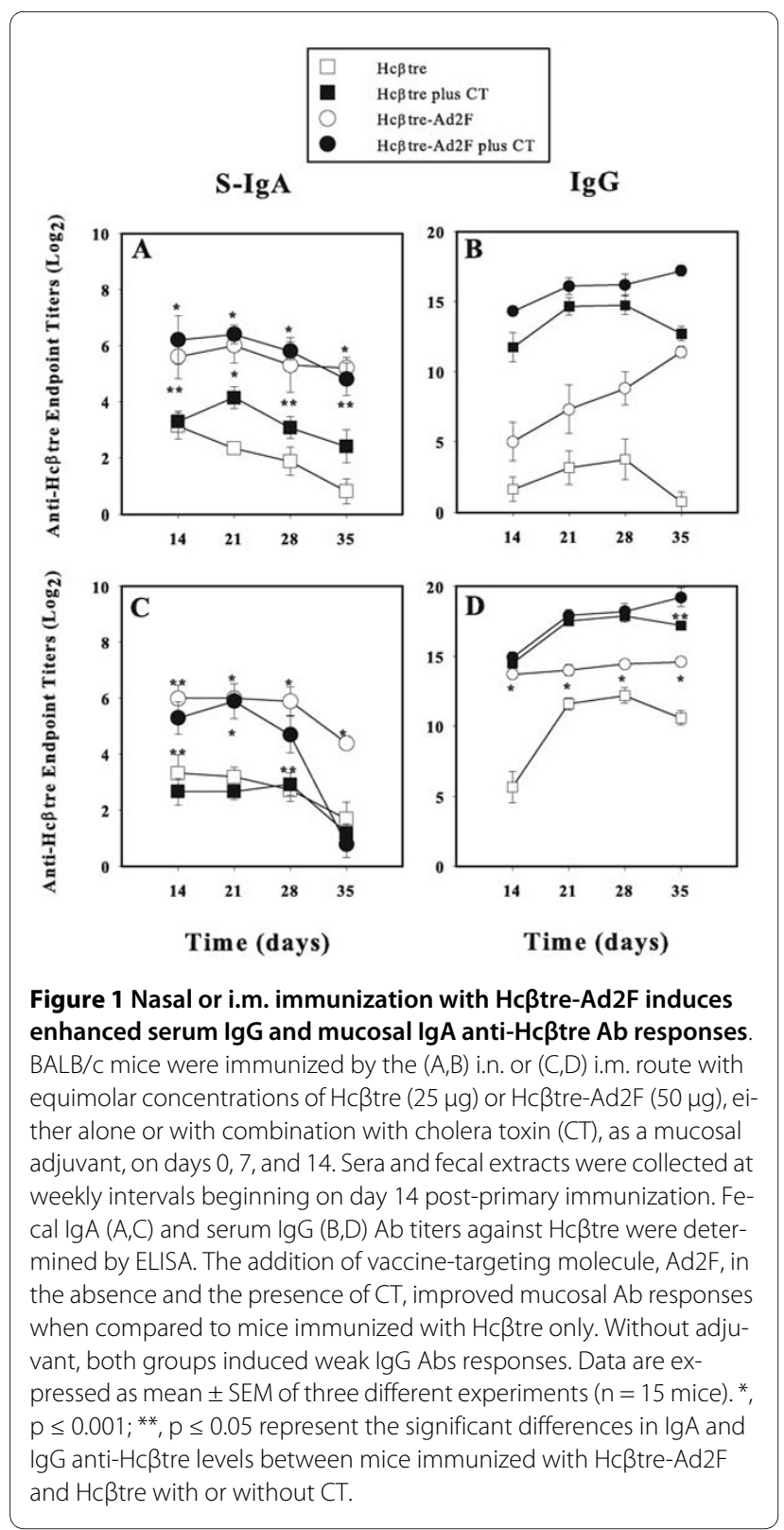

cant elevations $(\mathrm{p} \leq 0.001)$ in both IgG1 and IgG2a antiHc $\beta$ tre responses compared to the i.m. Hc $\beta$ tre plus CTimmunized mice, and both groups showed equivalent IgG2b responses (Figure $2 \mathrm{C}$ ). These results clearly show that $\mathrm{Hc} \beta$ tre evokes both mucosal and systemic immune $\mathrm{Ab}$ responses influenced by route of immunization, and the Ab titers are significantly improved when $\mathrm{Hc} \beta$ tre is genetically fused to Ad2F.

\section{Distribution of immune B cells following i.n. or i.m. immunization}

To learn the source of the immune Abs obtained upon immunization with $\mathrm{Hc} \beta$ tre-based vaccines, Ag-specific B cell responses from the various mucosal and systemic

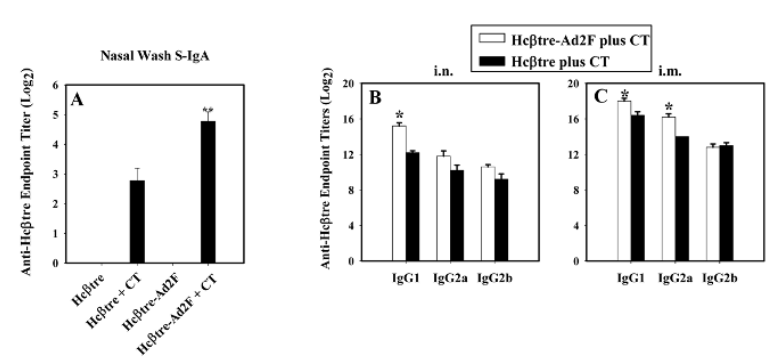

Figure 2 Nasal immunization with $\mathrm{Hc} \beta$ tre-Ad2F plus $\mathrm{CT}$ stimulates an enhanced nasal wash secretory (S)-IgA and elevated serum IgG1 and IgG2a anti-Hc $\beta$ tre $A b$ titers. (A) BALB/c mice were nasally immunized with $\mathrm{Hc} \beta$ tre-Ad2F or $\mathrm{Hc} \beta$ tre plus $C T$, as described in Figure. 1. Three weeks after final immunization, S-IgA Ab levels in nasal washes were determined by ELISA. Mice vaccinated nasally with $\mathrm{Hc} \beta$ tre-Ad2F + CT showed significantly greater levels of $\mathrm{Hc} \beta$ tre-specific S-IgA when compared with mice nasally dosed with $\mathrm{Hc} \beta$ tre $+\mathrm{CT}$. Data represent the mean \pm SEM $\left(n=15\right.$ mice). ${ }^{*}, p \leq 0.001$ represent the statistical differences in $\mathrm{Hc} \beta$ tre-specific S-IgA Abs between mice immunized with $\mathrm{Hc} \beta$ tre-Ad2F $+C T$ vs. mice immunized with $\mathrm{Hc} \beta$ tre + CT. Serum IgG subclass anti-Hc $\beta$ tre Ab responses on day 35 from (B) i.n. and (C) i.m. immunized mice ( $n=7$ mice/group) with $\mathrm{Hc} \beta$ tre-Ad2F or $\mathrm{Hc} \beta$ tre plus $C T$ were evaluated. Significant elevations in $(B, C) \lg G 1$ and (C) IgG2a Ab titers were observed by the $\mathrm{Hc} \beta$ tre-Ad2F plus $\mathrm{CT}$-immunized mice versus the $\mathrm{Hc} \beta$ tre plus CT-immunized mice: ${ }^{*}, p \leq 0.001$.

lymphoid tissues were measured using an Ab ELISPOT assay. Ag-specific Ab-forming cells (AFCs) were minimally detected in lymphoid tissues of mice vaccinated without CT (data not shown). Elevated Hc $\beta$ tre-specific IgA AFCs were detected in nasal-associated lymphoreticular tissue (NALT) $(\mathrm{p} \leq 0.001)$ and nasal passages (NPs) $(\mathrm{p} \leq 0.05)$ from mice i.n. immunized with Hc $\beta$ tre-Ad2F plus CT when compared to mice i.n. immunized with adjuvanted Hc $\beta$ tre (Figure $3 \mathrm{~A})$. The numbers of Ag-specific IgA AFCs in head and neck LNs (HNLNs), submaxillary glands (SMGs), spleens, Peyer's patches (PPs), and intestinal lamina propria (iLP) were similar to mice immunized with either Hc $\beta$ tre-Ad2F or Hc $\beta$ tre (Figure $3 A)$. Only minimal Ag-specific IgA AFCs were detected in mice i.m. immunized with our Hc $\beta$ tre-based vaccines (Figure 4A). Analysis of Ag-specific IgG AFC responses in i.n.-dosed mice exhibited elevated AFCs in HNLNs, SMG, spleen, and PPs $(\mathrm{p} \leq 0.05)$ of mice that received adjuvanted $\mathrm{Hc} \beta$ tre-Ad2F when compared with mice given $\mathrm{Hc} \beta$ tre (Figure $3 \mathrm{C}$ ). The presence of $\mathrm{Hc} \beta$ tre-specific IgG AFCs was detected in NALT and NPs in both groups of i.n.-vaccinated mice, but these were not statistically significant (Figure 3C). Mice i.m. immunized with $\mathrm{Hc} \beta$ tre-Ad2F plus CT showed increased levels of Ag-specific IgG AFCs in the peripheral LNs (PLNs), PPs ( $\mathrm{p} \leq$ $0.001)$, and HNLNs $(\mathrm{p} \leq 0.05)$ in contrast to mice that received Hc $\beta$ tre plus CT (Figure $4 \mathrm{C}$ ). Minimal IgA AFC responses were detected in any of the lymphoid tissues examined (Figure 4A). From these studies, we showed 


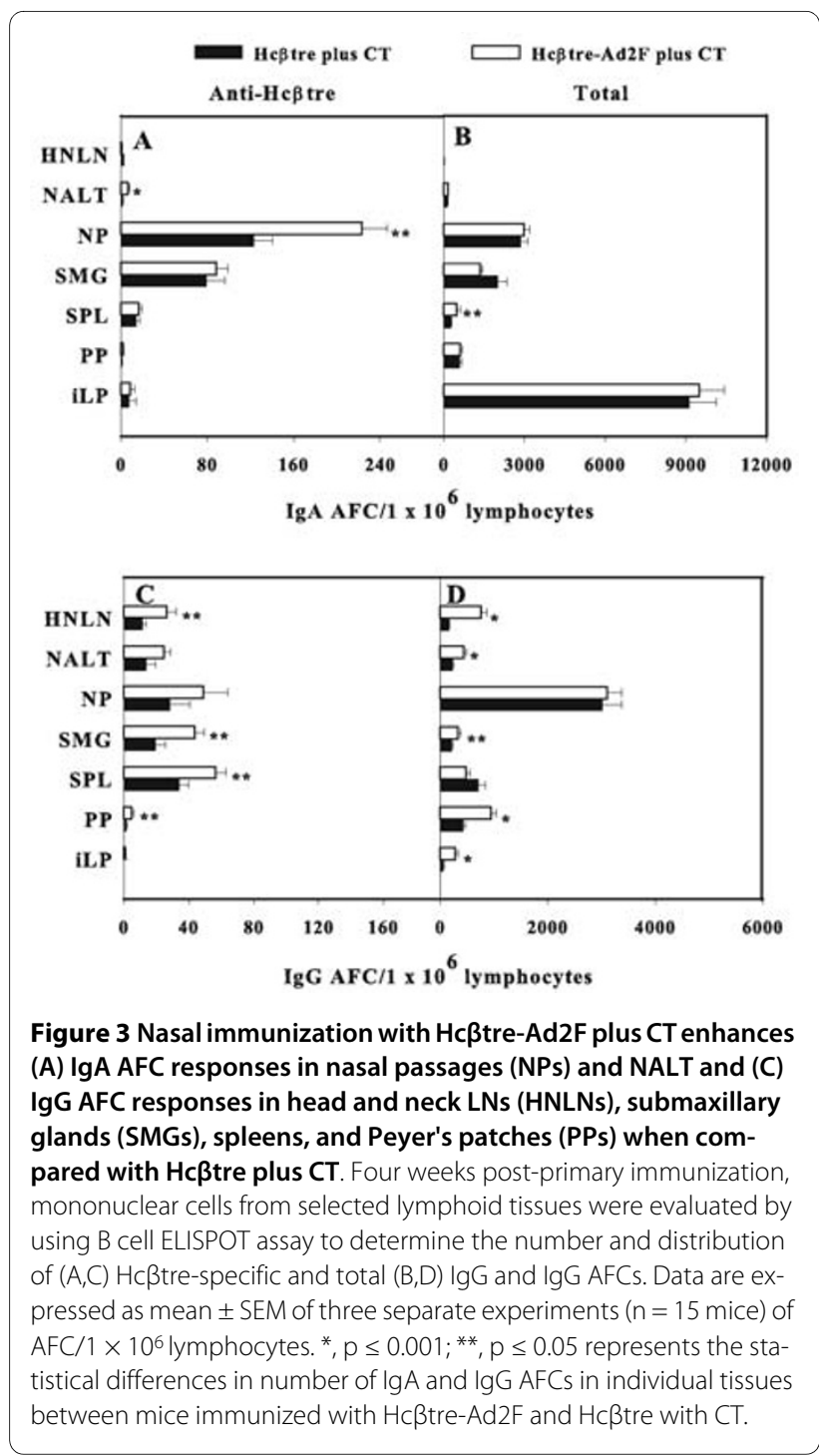

that nasal immunization with adjuvanted $\mathrm{Hc} \beta$ tre-Ad2F enhances IgA AFC responses in NP and NALT and IgG AFC responses in HNLNs, SMG, spleen, and PPs when compared with adjuvanted $\mathrm{Hc} \beta$ tre. In addition, these data show that i.m. immunization with $\mathrm{Hc} \beta$ tre-Ad2F in presence of CT enhances the number of IgG AFCs in PLNs, HNLNs, and PPs when compared to mice immunized with $\mathrm{Hc} \beta$ tre plus CT.

\section{Hc $\beta$ tre-based vaccines elicit a mixed Th cell response}

Since immunization with our $\mathrm{Hc} \beta$ tre-based vaccines induced elevated Ag-specific $\mathrm{Ab}$ responses in both mucosal and systemic tissues, it was important to examine the nature of Th cells that support these $\mathrm{Hc} \beta$ tre-specific B cell responses. Splenic, HNLN, and mesenteric LN (MLN) lymphocytes from mice dosed with adjuvanted Hc $\beta$ tre and $\mathrm{Hc} \beta$ tre-Ad2F vaccines were restimulated with $\mathrm{Hc} \beta$ tre and subsequently evaluated for the produc-

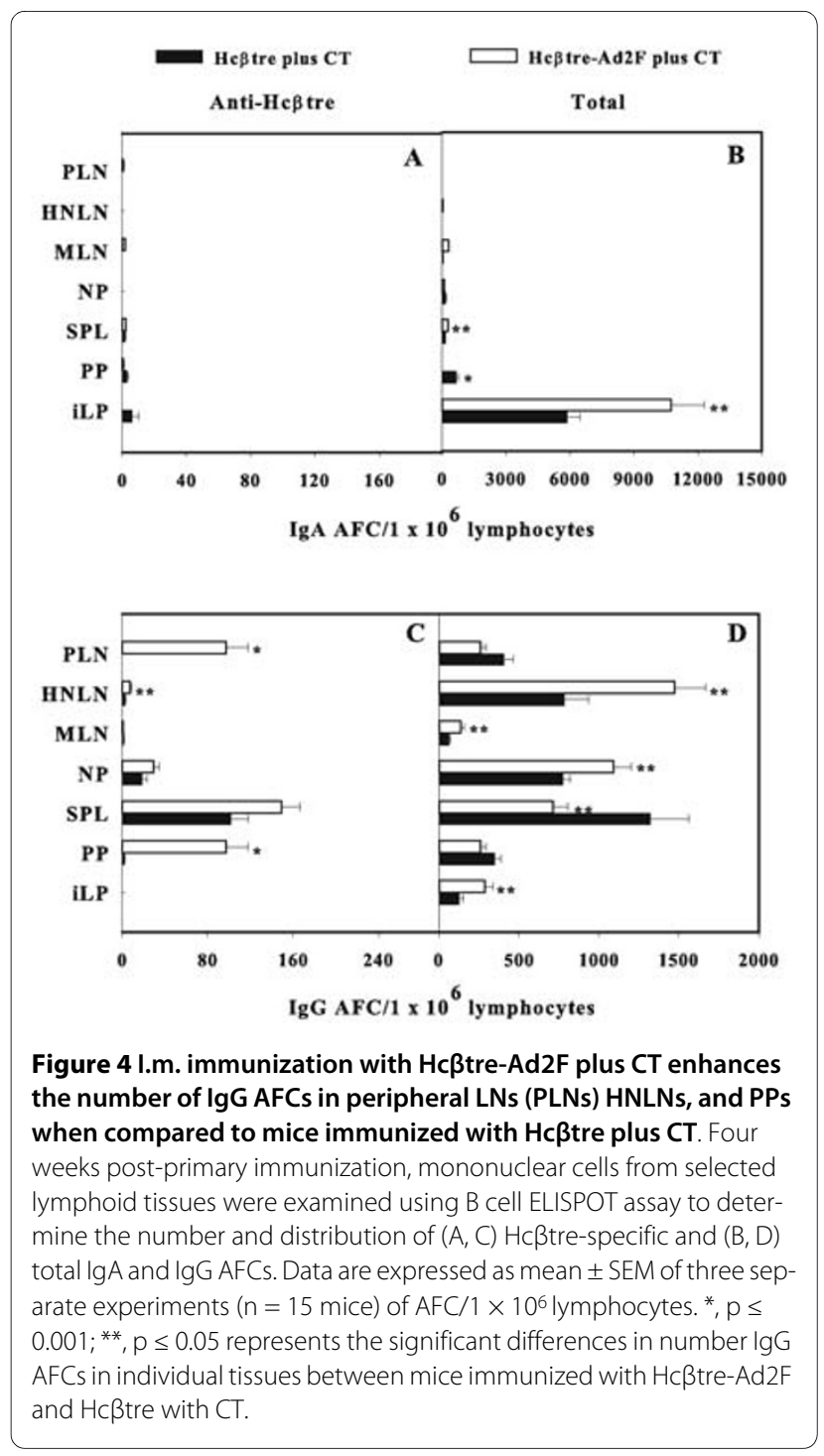

tion of IFN- $\gamma$, IL-4, IL-5, IL-10, and IL-13 by cytokine ELISPOT method. Analysis of Th1 and Th2 cytokine production by Ag-stimulated $\mathrm{T}$ cells revealed a varied Th cell phenotype. For i.n.-immunized mice, splenic IL-5 and IL-10 cytokine-forming cells (CFCs) were induced to similar levels for both vaccine groups, but increased IFN$\gamma$ and IL-13 CFCs were observed in Hc $\beta$ tre-immunized mice and IL-4 in Hc $\beta$ tre-Ad2F-dosed mice (Figure $5 \mathrm{~A}$ ). In the HNLNs, the IFN- $\gamma$, IL-4, IL-5, and IL-13 were particularly enhanced in $\mathrm{Hc} \beta$ tre-vaccinated mice (Figure 5B). In the MLNs, the levels of IL-4, IL-5, and IL-10 CFCs were significantly greater than in $\mathrm{Hc} \beta$ tre-Ad2F-dosed mice (Figure $5 \mathrm{C}$ ). For i.m.-immunized mice, splenic IFN$\gamma$, IL-4, IL-5, and IL-10 CFCs (Figure 6A), HNLN IL-4, IL5, IL-10, and IL-13 CFCs (Figure 6B), and MLN IFN- $\gamma$,IL4, and IL-5 CFCs (Figure 6C) were significantly elevated in the $\mathrm{Hc} \beta$ tre-Ad2F-immunized group when compared to $\mathrm{Hc} \beta$ tre-immunized mice. These results showed that 


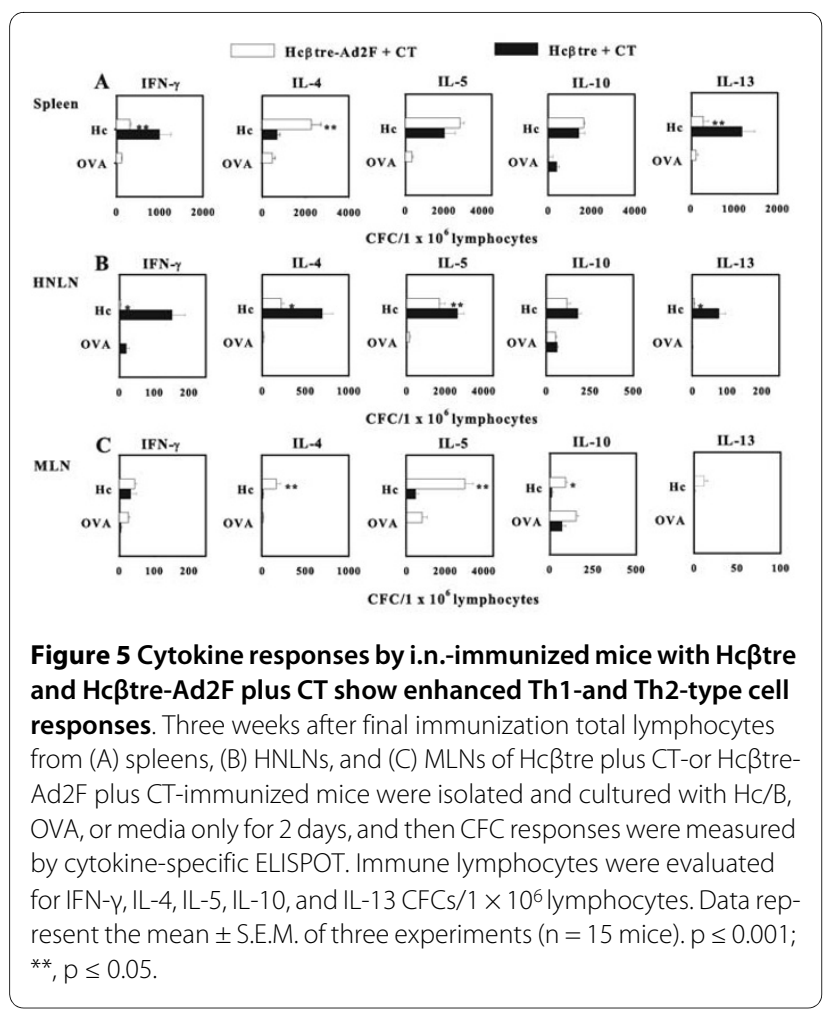

nasal immunization with adjuvanted Hc $\beta$ tre-Ad2F elicited an enhanced Th2 cell bias as opposed to mice immunized with Hc $\beta$ tre plus CT that elicited a mixed Th cell response. I.m. immunization with either vaccine com-

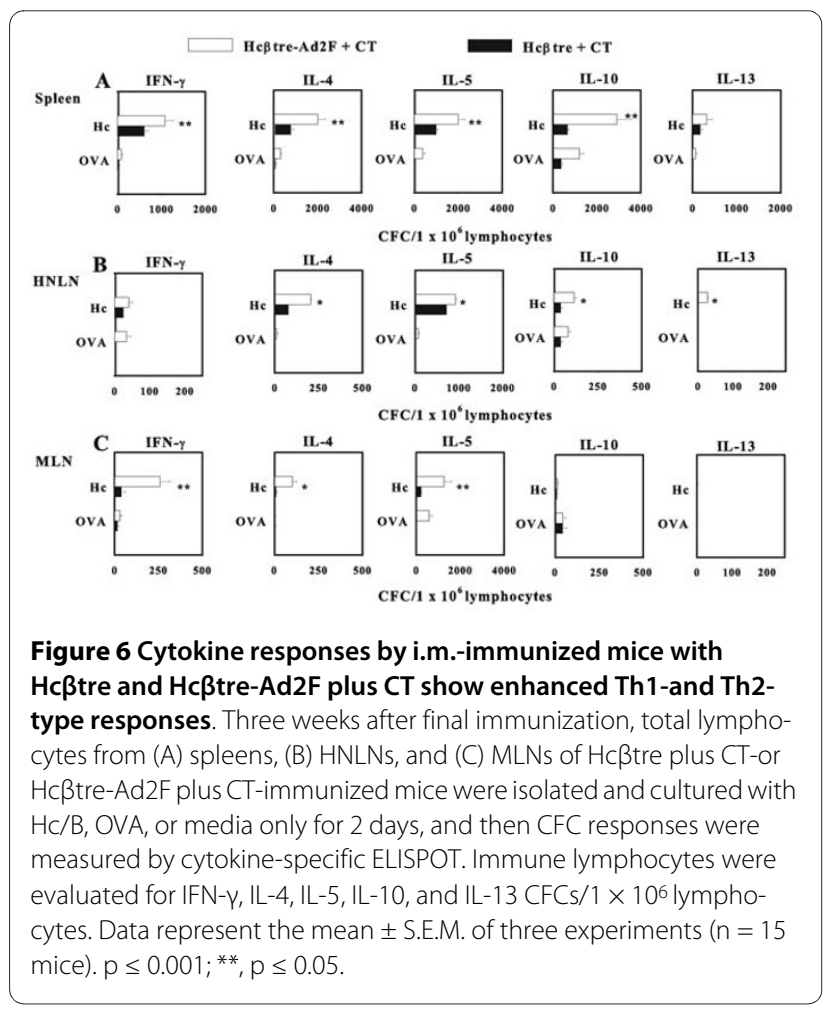

bined with adjuvant showed mixed Th cell responses. For either immunization route, it was evident that the IgG1 $\mathrm{Ab}$ responses were supported by elevations in Th2 cell cytokines and IgG2a Ab responses supported by IFN- $\gamma$.

\section{Mouse neutralization assay}

To evaluate the protective Ab responses, serum samples from the various i.n. immunization groups were tested for their ability to neutralize native BoNT/B. Three sources of sera were assessed: (i) sera from unimmunized mice; (ii) sera from mice vaccinated with $\mathrm{Hc} \beta$ tre plus CT; and (iii) sera from mice vaccinated with $\mathrm{Hc} \beta$ tre-Ad2F plus CT. Mice were immunized four times on days 0, 7, 14 , and 21 , and serum samples were collected on day 57 post-primary immunization, at which time endpoint titers were determined for $\mathrm{Hc} / \mathrm{B}$. Mice given either $\mathrm{Hc} \beta$ tre or Hc $\beta$ tre-Ad2F showed elevated serum IgG responses against $\mathrm{Hc} / \mathrm{B}$, while naive sera showed no reactivity (Table 1). Sera samples were incubated with 2.0 $\mathrm{LD}_{50}$ of the BoNT/B at room temperature for $60 \mathrm{~min}$ before i.p. injection into naïve BALB/c mice. All mice given naive sera succumbed to intoxication by $24 \mathrm{~h}$ (Table 1). Mice given BoNT/B plus pooled sera from mice nasally immunized with either adjuvanted $\mathrm{Hc} \beta$ tre or Hc $\beta$ tre-Ad2F survived. These studies demonstrated that immunization with $\mathrm{Hc} \beta$ tre-Ad2F or $\mathrm{Hc} \beta$ tre could elicit neutralizing Abs that protect against systemic challenge.

\section{Discussion}

Botulism results from absorption of the toxin into the systemic circulation from either a mucosal surface or a wound. BoNT intoxication is typically attributed to oral ingestion of contaminated food either with the toxin or bacteria producing the toxin [6]. Although natural exposure via the inhalational route is unlikely [18], illicit aerosol releases have occurred [28]; thus, there is a

\section{Table 1: 24 h Survival status in BoNT/B-mouse} neutralization assaya.

\begin{tabular}{cccc}
\hline Vaccination Group $^{\mathbf{b}}$ & Sera Titerc & \%Survivald & p-value $^{\mathbf{e}}$ \\
\hline Naïve & 0 & 0 & - \\
Hc $\beta$ tre/B + CT & $19.5 \pm 0.5$ & 100 & $<0.05$ \\
\hline Hc $\beta$ tre-Ad2F + CT & $19.8 \pm 0.2$ & 100 & $<0.05$ \\
\hline
\end{tabular}

a Pooled sera from nasally vaccinated or naive mice incubated for $1 \mathrm{~h}$ with 2.0

$\mathrm{LD}_{50}$ of BoNT/B in PBS- $0.2 \%$ gelatin and then injected into naive

mice.

b Source of sera used.

c Endpoint titer for $\mathrm{Hc} / \mathrm{B}$ in pooled sera (10 mice/group).

d Survival of BoNT/B-challenged mice after $24 \mathrm{~h}$.

e $p$-value determined by Kaplan-Meier method. 
precedence to stimulate mucosal immune responses to aid in conferring protection by neutralizing the toxin at the mucosal surface. While most studies have focused on BoNT/A [13,29,30], few studies have evaluated BoNT/Bbased vaccines [31,32]. Since BoNT/B is often implicated in human illness, the present study focuses on generating a protective mucosal vaccine.

Efforts to develop an efficacious vaccine for botulism are warranted because the current pentavalent toxoid vaccine has a number of disadvantages [23], including the limited ability to stimulate mucosal Abs when given parenterally [30] and the required yearly boosts to maintain protective Ab levels [13]. To address these shortcomings, recombinant vaccine approaches would best preserve neutralization epitopes, and consideration of mucosal immunization approaches may best serve to induce both mucosal and systemic immunity. Immunization of both mucosal and systemic immune compartments could then facilitate the clearance of the toxin from tissues and inhibit its absorption to mucosal surfaces $[31,33]$.

Current efforts to develop BoNT vaccines have focused primarily on immunizing with recombinant Hc polypeptides capable of evoking protective immunity against BoNT challenge $[10,12,34,35]$. Hc is an efficient immunogen that combined with the ability to penetrate the epithelial membranes raises the possibility that the Hc can serve as a mucosal vaccine against botulism. Furthermore, as previously shown for protection against BoNT/ A [23] and suggested by others [36], the entire Hc fragment is not required for protection and can be further reduced to contain the $\mathrm{C}$-terminal $\beta$-trefoil subdomain. Our previous study provided the first evidence the $\mathrm{Hc} \beta$ tre fragment retains its immunogenicity and protective capacity when delivered nasally [23]. In addition, since $\beta$-trefoil structure is conserved among the seven BoNTs, it is plausible this conserved structure can evoke cross-reactive Abs against the other serotypes. In addition, we sought to develop a second animal model for BoNT intoxication using antisera developed in rabbits. When testing commercially prepared Hc against Hc $\beta$ tre/ A-Ad2F in rabbits formulated with $\mathrm{CT}$ or a non-toxin adjuvant, we found the $\mathrm{Hc} \beta$ tre/A-Ad2F vaccine was superior to $\mathrm{Hc}$ in conferring protection against BoNT/A challenge in a mouse neutralization assay when using antisera developed in rabbits (manuscript in preparation). These results suggest that the Hcßtre-Ad2F immunogen is effective in eliciting BoNT-specific Abs.

While next generation vaccines may significantly improve vaccine efficacy, these are often poorly immunogenic when applied mucosally [33]. To enhance their immunogenicity, vaccines would require an effective adjuvant to bolster protective immunity. Currently, a major obstacle to adapting mucosal vaccines for humans is the lack of a mucosal adjuvant suitable for use in humans. Nasal application of adenovirus-based vaccines offers one approach to stimulate robust systemic and mucosal $\mathrm{Ab}$ responses, as well as cell-mediated immunity [25]. In fact, recombinant adenovirus bearing the $\mathrm{Hc}$ transgene has been shown to effectively stimulate neutralizing Abs to BoNT/C [37]. However, one concern limiting the use of adenoviral vaccine vectors is that preexisting immunity to adenoviruses induced by natural exposure can potentially neutralize adenoviral vaccines via reactivity against its hexon protein [24], thus, preventing the development of a protective immune response [38]. Admittedly, studies have shown that i.n. immunization seems to avoid the stimulation of systemic neutralizing Abs [39]. However, another concern is the possible retrograde uptake of replication-deficient adenovirus vectors into the olfactory bulb [24], suggesting perhaps a natural consequence of wild-type Ad infection. Although adenoviruses have been found in the CNS, they are not generally considered as "neurotropic" and isolation of them from brain tissues is rare [40]

To circumvent such concerns, efforts were focused on developing a targeting moiety to enable mucosal vaccination based upon the adenovirus adhesin or $\mathrm{F}$ protein instead of the whole virus. In support of our previous study against BoNT/A [23], our current results clearly demonstrated that the inclusion of the targeting molecule, Ad2F, also boosts the levels of mucosal IgA and systemic IgG Abs against serotype B when administrated mucosally (Figure 1A,B). Moreover, it is important to emphasize that in the absence of $\mathrm{CT}$ adjuvant, nasal immunization with our recombinant $\mathrm{Ad} 2 \mathrm{~F}$ fusion vaccine stimulated similar levels of fecal IgA Abs as in mice coimmunized with CT (Figure 1A). In the absence of the Ad2F targeting moiety, Hc $\beta$ tre induced only a weak mucosal $\mathrm{Ab}$ response even with $\mathrm{CT}$. These findings suggest perhaps Ad2F possesses adjuvant-promoting properties, which, in turn, could limit the use of adjuvants and diminish the number of immunizations required to stimulate protective immunity. Current studies are addressing such possibilities. Interestingly, our study showed that mice given our fusion vaccine via the i.m. route produced elevated levels of both mucosal IgA and systemic IgG Abs (Figure 1C,D). Notably, i.m. immunization with Hc $\beta$ treAd2F in the absence of CT showed elevated IgG Ab titers that remained elevated for at least 35 days post-primary immunization. One possible explanation for our findings could be attributed to the interactions between Ad2F and the coxsackie adenovirus receptor, which is expressed in a wide range of cell types. This interaction may allow more effective delivery of the vaccine into the cells. In addition, similar to our previous study [23], the Hc $\beta$ tre's immunogenicity was markedly improved by the addition of Ad2F (Figures 1, 2, 3 and 4). However, only a low level of mucosal Abs after day 35 was observed (Figure 1C), sug- 
gesting that long term mucosal memory was not induced when the Ad2F-based vaccine was given parenterally. Since very few IgA AFCs were detected in any of the lymphoid tissues of i.m.-immunized mice (Figure 4A), such evidence suggests that transient IgA Ab responses were induced during immunization.

Few studies have evaluated whether Th1 or Th2 cells are beneficial for optimal Ab production for the Hc-based vaccines. A recent study by Kobayashi et al. [30] suggests that BoNT/A-specific Abs induced by mucosal BoNToxoid immunization are mediated via Th2-type cytokines. Herein this report, a combination of Th cell types supports the BoNT/B-specific Ab responses (Figures 5 and 6). Notable enhancements in Th2-type cells were detected in the spleens and MLNs of i.n. Hcßtre-Ad2F plus CT-immunized mice (Figure $5 \mathrm{~A}, \mathrm{C}$ ), and likewise for i.m. Hcßtre-Ad2F plus CT-immunized mice (Figure $6 \mathrm{~A}, \mathrm{C})$ suggesting these responses supported the observed, elevated IgG1 Ab responses (Figure 2B,C). These findings further corroborate the results of our previous study showing that the $\mathrm{Hc} \beta$ tre/A vaccine for BoNT/A also elicits a mixed Th cell response [23].

Nasal administration of $\mathrm{Hc} \beta$ tre-based vaccines induced significant systemic and mucosal Abs. To assess their protective efficacy, a mouse neutralization assay, was performed (Table 1). Our data showed that passively administered sera from animals immunized with Hc $\beta$ tre or with Hc $\beta$ tre-Ad2F protected $100 \%$ mice for $24 \mathrm{hr}$ against $2.0 \mathrm{LD}_{50}$, suggesting neutralizing Abs are induced.

\section{Conclusion}

As previously shown for Hc $\beta$ tre/A [23], the Hc $\beta$ tre/B is also very immunogenic capable of eliciting elevated serum IgG and mucosal IgA Abs, especially when genetically fused to the adhesin, Ad2F. These enhanced Ab responses are supported by mostly by Th2 cell responses when $\mathrm{Hc} \beta$ tre-Ad2F is given nasally, and a mixed Th1/Th2 cell response is obtained subsequent i.m. immunization. The induced Abs were able to neutralize BoNT/B as evident in the mouse neutralization assay. Thus, these studies demonstrate that the Hc $\beta$ tre immunogen can be used as a BoNT vaccine.

\section{Methods}

\section{Protein expression and purification}

The strategy for the construction and expression of the recombinant proteins in Pichia pastoris was similar to those previously described [23]. A synthetic gene encoding for Hc/B amino acids E786 to E1291 (Genebank ID 6030102) was designed for expression $P$. pastoris taking into account the yeast $P$. pastoris taking into account the yeast codon bias, the reduction of $C$. botulinum A/T content, and the necessity for not depleting any particular tRNA pools. The Hc $\beta$ tre/B spans from position E1071 to
E1291, as predicted by others [22]. To clone the Hcßtre domain, we included seven more amino acids upstream from the predicted Hc $\beta$ tre beginning with E1071 to E1291 to facilitate proper folding of the relevant domain.

To clone the Hc/B gene into the expression vector, the synthetic gene encoding for $\mathrm{Hc} / \mathrm{B}$ was amplified with primers containing EcoRI and KpnI restriction sites. Likewise, to clone the Hcßtre, this gene was amplified from the synthetic Hc/B using primers containing EcoRI and $\mathrm{KpnI}$ restriction sites. In both cases, the $5^{\prime}$ primers containing the EcoRI site also provided an ATG initiation codon embedded into an optimal Kozak's sequence. The PCR products were cloned into a pCR2.1 TOPO TA cloning vector (Invitrogen Corp., Carlsbad, CA), excised with EcoRI and KpnI, and cloned into the P. pastoris expression vector pPICZ B cut with EcoRI and KpnI. Such a vector was designed to provide a $\mathrm{C}$-terminal histidine tag for subsequent protein purification. Ad2 fiber was amplified from genomic Ad2 DNA, as previously described [23], expressing only the C-terminal domain, G378 to E582, to include a short stretch of amino acids rich in Gly, the region containing the trimerizing domain and the region containing the globular knob domain, which is important for interacting with the coxsackievirus/adenovirus receptor on the cell surface [41].

To generate the $\mathrm{Hc} \beta$ tre/B-Ad2F, Hc $\beta$ tre was amplified with primers generating EcoRI and SalI ends cloned and excised with EcoRI and SalI. The mentioned region of Ad2F was amplified with primer generating SalI and KpnI ends, cloned, and excised with the corresponding enzymes. The vector pPICZ B was cut with EcoRI and KpnI. Finally, Hc $\beta$ tre-Ad2F was assembled via a tri-partite ligation. Primers were designed to allow in-frame junction between the different components and the formation of a flexible joint between Hc 3 tre and the Ad2F. These constructs were transformed into P. pastoris by electroporation.

Recombinant proteins in $P$. pastoris were purified following standard manufacturer's protocols. Following culture in YNB-methanol for 36-48 hr, cells were then harvested by centrifugation, and the pelleted biomass was disrupted with a bead-beater in ice. Debris was cleared by centrifugation, followed by filtration through a $1.2 \mu \mathrm{m}$ pre filter, then by filtration through a $0.45 \mu \mathrm{m}$ filter under vacuum. The cleared supernatant was then applied to a Talon column (BD Biosciences), as per the manufacturer's instruction. Purified proteins were eluted, titrated, and loaded onto a $12 \%$ polyacrylamide gel. Recombinant Hc $\beta$ tre proteins were analyzed by Coomassie-stained SDS-PAGE to assess the quality of the protein.

\section{Immunization}

BALB/c mice (National Cancer Institute) between 6 and 8 weeks old were maintained in the Montana State Univer- 
sity Animal Resources Center under pathogenic free conditions in individually ventilated cages under HEPAfiltered barrier conditions; sterile food and water were provided ad libitum. The mice were free of bacterial and viral pathogens, as determined by $\mathrm{Ab}$ screening and histopathologic analysis of major organs and tissues. All animal care and procedures were in accordance with institutional policies for animal health and well-being and approved by MSU Institutional Animal Care and Use Committee.

Vaccines were administered by the intranasal (i.n.) or intramuscular (i.m.) route. For i.n. immunization, mice were given equimolar amounts of Ag corresponding to 25 $\mu \mathrm{g}$ of $\mathrm{Hc} \beta$ tre or $50 \mu \mathrm{g}$ of $\mathrm{Hc} \beta$ tre-Ad2F and, in some instances as stated, given with $5 \mu \mathrm{g}$ of cholera toxin (CT; List Biological Laboratories), as indicated, and boosted with their respective vaccines on days 7 and 14 after initial immunization with $2 \mu \mathrm{g}$ of CT. For i.m. immunization, mice received $25 \mu \mathrm{g}$ of $\mathrm{Hc} \beta$ tre or $50 \mu \mathrm{g}$ of $\mathrm{Hc} \beta$ treAd2F with or without $1 \mu \mathrm{g}$ of CT on days 0,7 , and 14 .

\section{Measurement of anti-Hc $\beta$ tre Abs titers by ELISA}

Endpoint Ab titers in serum, fecal extracts, and nasal washes generated to $\mathrm{Hc} \beta$ tre/B were determined by the standard ELISA methods [23]. Serum and fecal samples were collected weekly; nasal washes, on day 35 upon study termination, from each mouse were assessed for their $\mathrm{Hc} \beta$ tre-specific Ab activity. Fecal extractions and nasal washes were performed, as previously described [23]. Flat-bottom, 96-wells (MaxiSorb, Nunc) were coated with recombinant $\mathrm{Hc} \beta$ tre $/ \mathrm{B}(5 \mu \mathrm{g} / \mathrm{ml})$ and incubated at $4^{\circ} \mathrm{C}$ overnight, followed by washing with phosphate-buffered saline (PBS) containing $0.05 \%$ Tween 20 (wash buffer). The plates were blocked with $1 \%$ bovine serum albumin (BSA) in PBS for $1 \mathrm{~h}$ at $37^{\circ} \mathrm{C}$. After washes, serial dilutions of serum or fecal samples in PBS containing 0.05\% Tween 20 and 0.5\% BSA were added to the plates and incubated at $4^{\circ} \mathrm{C}$ overnight. IgG, IgG subclasses, and IgA titers were determined using peroxidase-conjugated goat anti-mouse IgG, IgG1, IgG2a, IgG2b, or IgA (Southern Biotechnology Associates). Following $90 \mathrm{~min}$ of incubation at $37^{\circ} \mathrm{C}$ and the washing step, 2,2'-azinobis(3ethylbenthiazoline-6-sulfonic acid; Moss) was added as a substrate, and the plates were incubated for an additional $60 \mathrm{~min}$ at $25^{\circ} \mathrm{C}$. The endpoint titers were the reciprocal dilutions of the last dilution yielding an absorbance at $\mathrm{OD}_{415}$ above $0.100 \mathrm{OD}$ units above negative controls.

\section{Lymphocyte isolation}

Lymphocytes were isolated from nasal passages (NPs), submaxillary glands (SMGs), nasal-associated lymphoreticular tissue (NALT), small intestinal lamina propria (iLP), Peyer's patches (PPs), mesenteric lymph nodes (MLNs), spleens, peripheral LNs (PLNs), and head and neck LNs (HNLNs). PPs, MLNs, spleens, PLNs, NALT, and HNLNs were subjected to dounce homogenization, and resulting cell suspensions were filtered through Nitex (Fairview Fabrics), washed with complete medium (CM), and centrifuged. Mononuclear cells from NPs, SMGs, and iLP were isolated, as previously described [42]. Lymphocyte viability was $>95 \%$ from each tissue, as determined by trypan blue exclusion.

\section{Ab ELISPOT}

The $\mathrm{B}$ cell ELISPOT assay was used to quantify the numbers of IgA and IgG Ag-specific Ab-forming cells (AFCs). Mixed cellulose ester membrane-bottomed microtiter plates (MultiScreen-HA; Millipore) were coated at 100 $\mu \mathrm{l} /$ well with sterile PBS containing $5 \mu \mathrm{g} / \mathrm{ml}$ of recombinant $\mathrm{Hc} / \mathrm{B}$. For total IgA or IgG AFC determinations, wells were coated with $5 \mu \mathrm{g} / \mathrm{ml}$ goat anti-mouse IgG or IgA Abs (Southern Biotechnology Associates) in sterile PBS and incubated overnight at $25^{\circ} \mathrm{C}$. The plates were blocked with $200 \mu \mathrm{l} /$ well of RPMI 1640 medium supplemented with $10 \%$ FBS (RPMI-FBS) for $1 \mathrm{~h}$ at $37^{\circ} \mathrm{C}$. Blocking medium was discarded, and $100 \mu \mathrm{l}$ of cells from each tissue at varying concentrations were added to each well. After incubation overnight at $37^{\circ} \mathrm{C}$ in a humidified chamber under $5 \% \mathrm{CO}_{2}$, the plates were washed with PBS containing $0.05 \%$ Tween-20 followed by overnight incubation with $100 \mu \mathrm{l} /$ well of $1.0 \mu \mathrm{g} / \mathrm{ml} \mathrm{HRP} \mathrm{conjugates} \mathrm{of} \mathrm{goat} \mathrm{anti-}$ mouse IgG or IgA Abs (Southern Biotechnology Associates). Wells were washed, and substrate solution containing 3-amino-9-ethyl-carbazole was added. After 20-30 min, the reaction was stopped by rinsing water. AFCs were enumerated by counting using a dissection microscope (Leica).

\section{Cytokine ELISPOT}

Total splenic, HNLN, and MLN mononuclear cells $(5 \times$ $10^{6} / \mathrm{ml}$ ) were resuspended in CM and restimulated with $10 \mu \mathrm{g} / \mathrm{ml}$ recombinant $\mathrm{Hc} / \mathrm{B}$, OVA (tissue-culture grade; Sigma-Aldrich), or media in the presence of $10 \mathrm{U} / \mathrm{ml}$ human IL-2 (PeproTech) for 2 days at $37^{\circ} \mathrm{C}$. Cells were washed and resuspended in $\mathrm{CM}$. Stimulated lymphocytes were then evaluated by IFN- $\gamma$, IL-4, IL-5, IL-10, and IL13-specific ELISPOT assays [23]. Cell suspensions were prepared at 2 different densities: $2.4 \times 10^{5}$ and $8 \times 10^{4}$ cells ml. ELISPOT plates (MultiScreen-HA; Millipore) were coated overnight with the desired anti-cytokine capture antibody at $25^{\circ} \mathrm{C}$. Antibodies were removed, and plates were blocked with $200 \mu \mathrm{l} /$ well of CM for $1 \mathrm{~h}$ at $37^{\circ} \mathrm{C}$. Cell suspensions were added in triplicate to each well, and the plates were allowed to incubate at $37^{\circ} \mathrm{C}, 5 \%$ $\mathrm{CO}_{2}$ for $24 \mathrm{~h}$. Plates were washed with PBS containing $0.05 \%$ Tween-20, and biotinylated antibodies for either IFN- $\gamma$, IL-4, IL-5, IL-10, or IL-13 (BD Pharmingen) were added, and plates were incubated overnight at $4^{\circ} \mathrm{C}$. After 
washing the wells, plates were incubated for $1 \mathrm{~h}$ with a peroxidase-labeled anti-biotin Ab (Vector Laboratories) at $25^{\circ} \mathrm{C}$. The plates were washed again and developed by incubating for $45 \mathrm{~min}$ with substrate solution containing 3-amino-9-ethyl-carbazole, followed by rinsing in tap water. Spots were counted using a dissection microscope (Leica).

\section{Mouse neutralization assay}

For the mouse neutralization assay, groups of mice were immunized on days $0,7,14$, and 21 with equimolar doses of $\mathrm{Hc} \beta$ tre or Hc $\beta$ tre-Ad2F with CT. Another group was left unimmunized. Ab titers against $\mathrm{Hc} \beta$ tre/B and $\mathrm{Hc} / \mathrm{B}$ were determined for all groups of mice. To assess the collected immune sera pooled from each vaccinated group, on day $57,50 \mu \mathrm{l}$ of pooled sera from each immunization group were diluted $1: 2$ in PBS containing $0.2 \%$ gelatin, incubated for $1 \mathrm{~h}$ at room temperature with 2.0 mouse $\mathrm{LD}_{50}$ of BoNT/B $\left(1.0 \times 10^{8} \mathrm{MLD}_{50} / \mathrm{mg}\right.$, Lot no. B04170901; Metabiologics), and then injected i.p. into five naïve $\mathrm{BALB} / \mathrm{c}$ mice. Mice were observed hourly for signs of BoNT intoxication, and when signs of neuromuscular weakness become obvious, animals were euthanized in accordance with AAALAC guidelines.

\section{Statistical Analysis}

To evaluate differences among variations in $A b$ titers, $A b$ AFCs, and cytokine CFCs, an ANOVA followed by Tukey's method was used and discerned to the 95 percent confidence interval. The Kaplan-Meier method (GraphPad Prism, GraphPad Software, Inc., San Diego, CA) was applied to obtain the survival fractions following intoxication with BoNT/B. Using the Mantel-Haenszel log rank test, the P-value for statistical differences among surviving BoNT/B challenge from sera obtained from $\mathrm{Hc} \beta$ tre plus CT-or Hc $\beta$ tre-Ad2F plus CT-vaccinated mice and naive mice were discerned at the 95 percent confidence interval.

\section{Authors' details}

${ }^{1}$ Veterinary Molecular Biology, Montana State University, Bozeman, MT 59717, USA

2 Department of Pathology, Duke University Medical Center, Durham, NC 27710, USA

\section{Abbreviations}

Ad2F: adenovirus 2 fiber protein; AFC: Ab-forming cell; BoNT: botulinum neurotoxin; BSA: bovine serum albumin; CFC: cytokine-forming cell; CM: complete medium; CT: cholera toxin; $\mathrm{Hc}$ : carboxyl terminal domain of heavy chain; Hc $\beta$ tre: $\beta$-trefoil domain of Hc for BoNT; HNLN: head and neck lymph node; iLP: intestinal lamina propria; i.n.: intranasal; i.m.: intramuscular; MLN: mesenteric lymph node; NALT: nasal-associated lymphoreticular tissue; NP: nasal passage; PLN: peripheral lymph node; PP: Peyer's patch; SMG: submaxillary gland.

\section{Competing interests}

The authors declare that they have no competing interests.

\section{Authors' contributions}

BC, MM, HFS, and DWP conceived and designed the experiments. BC performed the immune evaluation studies, and DWP conducted the mouse neutralization study. MM made the genetic constructs in yeast for $\mathrm{Hc}, \mathrm{Hc} \beta$ tre, and $\mathrm{Hc} \beta$ tre-Ad2F, and SG purified the recombinant proteins. BC, HFS, and DWP analyzed the data. BC and DWP prepared the manuscript, and all authors read approved the final manuscript.

\section{Acknowledgements}

This work was supported by Public Health Service grants Al-56286, Rocky Mountain Research Center of Excellence, NIH U54 Al-06537, and in part by Montana Agricultural Station and U.S. Department of Agriculture Formula Funds. The Veterinary Molecular Biology flow cytometry facility was in part supported by NIH/NCRR COBRE P20 RR-020185. The authors thank Ms. Nancy Kommers for her assistance in preparing this manuscript.

\section{Author Details}

Veterinary Molecular Biology, Montana State University, Bozeman, MT $59717-$ 3610, USA and 2Department of Pathology, Duke University Medical Center, Durham, North Carolina 27710, USA

Received: 6 January 2010 Accepted: 7 July 2010

Published: 7 July 2010

\section{References}

1. Hatheway CL: Toxigenic clostridia. Clin Microbiol Rev 1990, 3:66-98

2. Hatheway C: Clostridium botulinum. In Infectious Diseases Edited by: Bartlett JG, Blacklow NR. Orlando: WB Saunders; 1991:583-1586.

3. Villar RG, Elliott SP, Davenport KM: Botulism: the many faces of botulinum toxin and its potential for bioterrorism. Infect Dis Clin N Am 2006, 20:313-327.

4. Shukla HD, Sharma SK: Clostridium botulinum: a bug with beauty and weapon. Crit Rev Microbiol 2005, 31:11-18.

5. Taylor A: Botulism and its control. Am J Nursing 1973, 73:1380-1382

6. Horowitz B: Botulinum Toxin. Crit Care Clin 2005, 21:825-839.

7. Arnon SS, Schechter R, Inglesby TV, Henderson DA, Bartlett JG, Ascher MS, Eitzen E, Fine AD, Hauer J, Layton M, et al:: Botulism toxin as a biological weapon: medical and public health management. JAMA 2001, 285:1059-1070.

8. Shapiro RL, Hatheway C, Swerdlow DL: Botulism in the United States: a clinical and epidemiological review. Ann Intern Med 1998, 129:221-228.

9. Smith $L A$, Rusnak JM: Botulinum neurotoxin vaccines: past, present, and future. Crit Rev Immunol 2007, 27:303-318.

10. Byrne MP, Smith LA: Development of vaccines for prevention of botulism. Biochimie 2000, 82:955-966.

11. Byrne MP, Smith TJ, Montgomery VA, Smith LA: Purification, potency, and efficacy of the botulinum neurotoxin type $A$ binding domain from Pichia pastoris as a recombinant vaccine candidate. Infect Immun 1988, 66:4817-4822

12. Baldwin MR, Tepp WH, Przedpelski A, Pier CL, Bradshaw M, Johnson EA, Barbieri JT: Subunit vaccine against the seven serotypes of botulism. Infect Immun 2008, 76:1314-1318.

13. Clayton J, Middlebrook JL: Vaccination of mice with DNA encoding a large fragment of botulinum neurotoxin serotype A. Vaccine 2000, 18:1855-1862.

14. Yu Y-Z, Zhang S-M, Sun Z-W, Wang S, Yu W-Y: Enhanced immune responses using plasmid DNA replicon vaccine encoding the $\mathrm{Hc}$ domain of Clostridium botulinum neurotoxin serotype A. Vaccine 2007, 25:8843-8850

15. Montecucco C, Schiavo G: Mechanism of action of tetanus and botulinum neurotoxin. Mol Microbiol 1994, 13:1-8.

16. Zhou L, de Paiva A, Liu D, Aoki R, Dolly JO: Expression and purification of the light chain of botulinum neurotoxin $A$ : A single mutation abolishes its cleavage of SNAP-25 and neurotoxicity after reconstitution with the heavy chain. Biochemistry 1995, 34:1517-1518.

17. Smith LA: Development of recombinant vaccines for botulinum neurotoxin. Toxicon 1998, 36:1539-1548.

18. Park J-B, Simpson LL: Inhalational poisoning by botulinum toxin and inhalation vaccination with its heavy-chain component. Infect Immun 2003, 71:1147-1154 
19. Lacy DB, Tepp W, Cohen AC, DasGupta BR, Stevens RC: Crystal structure of botulinum neurotoxin type $A$ and implications for toxicity. Nat StruC Biol 1998, 5:898-902.

20. Swaminathan S, Eswaramoorthy S: Structural analysis of the catalytic and binding sites of Clostridium botulinum neurotoxin B. Nat Struct Biol 2000, 7:693-699.

21. Lebeda FJ, Olson MA: Secondary structural predictions for the clostridial neurotoxins. Proteins: Struct Funct Gen 1994, 20:293-300.

22. Ginalski K, Venclovas C, Lesyng B, Fidelis K: Structure-based sequence alignment for the $\beta$-trefoil subdomain of the clostridial neurotoxin family provides residue level information about the putative ganglioside binding site. FEBS Lett 2000, 482:119-124.

23. Maddaloni M, Staats HF, Mierzejewska D, Hoyt T, Robison A, Callis G, Kozaki S, Kiyono H, McGhee JR, Fujihashi K, Pascual DW: Mucosal vaccine targeting improves onset of mucosal and systemic immunity to botulinum neurotoxin A. J Immunol 2006, 177:5524-5532.

24. Tatsis N, Ertl HCJ: Adenoviruses as vaccine vectors. Mol Ther 2004, 10:616-629.

25. Santosuosso M, McCormick S, Xing Z: Adenoviral vectors for mucosal vaccination against infectious diseases. Viral Immunol 2005, 18:283-291.

26. Boyer JL, Kobinger G, Wilson JM, Crystal RG: Adenovirus-based genetic vaccines for biodefense. Hum Gene Therapy 2005, 16:157-168.

27. Bergelson JM, Cunningham JA, Droguett G, Kurt-Jones EA, Krithivas A, Hong JS, Horwitz MS, Crowell RL, Finberg RW: Isolation of a common receptor for Coxsackie $B$ viruses and adenoviruses 2 and 5 . Science 1997, 275:1320-1323.

28. Josko D: Botulin toxin: a weapon in terrorism. Clin Lab Sci 2004, 17:30-34.

29. Pier $C L$, Tepp WH, Bradshaw M, Johnson EA, Barbieri JT, Baldwin MR: Recombinant holotoxoid vaccine against botulism. Infect Immun 2008, 76:437-442

30. Kobayashi R, Kohda T, Kataoka K, Ihara H, Kozaki S, Pascual DW, Staats HF, Kiyono H, McGhee JR, Fujihashi K: A novel neurotoxoid vaccine prevents mucosal botulism. J Immunol 2005, 174:2190-2195.

31. Ravichandran E, Al-Saleem FH, Ancharski DM, Elias MD, Singh AK, Shamim $M$, Gong $Y$, Simpson LL: Trivalent vaccine against botulinum toxin serotypes $A, B$, and $E$ that can be administered by the mucosal route. Infect Immun 2007, 75:3043-3054.

32. Boles J, West M, Montgomery V, Tammariello R, Pitt ML, Gibbs P, Smith L, LeClaire RD: Recombinant $C$ fragment of botulinum neurotoxin $B$ serotype $\left(r B o N T B\left(H_{c}\right)\right.$ immune response and protection in the rhesus monkey. Toxicon 2006, 47:877-884.

33. Goyal AK, Khatri K, Mishra N, Vyas SP: New patents on mucosal delivery of vaccines. Expert Opin Ther Pat 2008, 18:1271-1288.

34. Clayton MA, Clayton JM, Brown DR, Middlebrook JL: Protective vaccination with a recombinant fragment of Clostridium botulinum neurotoxin serotype A expressed from a synthetic gene in Escherichia coli. Infect Immun 1995, 63:2738-2742.

35. Baldwin MR, Tepp WH, Pier CL, Bradshaw M, Ho M, Wilson BA, Fritz RB, Johnson EA, Barbieri JT: Characterization of the antibody response to the receptor binding domain of botulinum neurotoxin serotypes $A$ and E. Infect Immun 2005, 73:6998-7005.

36. Dertzbaugh MT, West MW: Mapping of protective and crossreactive domains of the type A neurotoxin of Clostridium botulinum. Vaccine 1996, 14:1538-1544

37. Xu Q, Pichichero ME, Simpson LL, Elias M, Smith LA, Zeng M: An adenoviral vector-based mucosal vaccine is effective in protection against botulism. Gene Ther 2009, 16:367-375.

38. Boyer JL, Kobinger G, Wilson JM, Crystal RG: Adenovirus-based genetic vaccines for biodefense. Hum Gene Therapy 2005, 16:157-168.

39. Muralidhar G, Navaratnam M, Englund N, Hariharan M, Sauer SL: Development of an adenoviral-based vaccine for HIV/AIDS. Cellscience Rev 2005, 2:1-15.

40. Soudais C, Laplace-Builhe C, Kissa K, Kremer EJ: Preferential transduction of neurons by canine adenovirus vectors and their efficient retrograde transport in vivo. FASEB J 2001, 12:2283-2285.

41. Barnett BG, Crews CJ, Douglas JT: Targeted adenoviral vectors. Biochem Biophys Acta 2002, 1575:1-14.

42. Csencsitis KL, Pascual DW: Absence of L-selectin delays mucosal B cell responses in non-intestinal effector tissues. J Immunol 2002, 169:5649-5659. doi: 10.1186/1471-2172-11-36

Cite this article as: Clapp et al., Adenovirus F protein as a delivery vehicle for botulinum B BMC Immunology 2010, 11:36

\section{Submit your next manuscript to BioMed Central and take full advantage of:}

- Convenient online submission

- Thorough peer review

- No space constraints or color figure charges

- Immediate publication on acceptance

- Inclusion in PubMed, CAS, Scopus and Google Scholar

- Research which is freely available for redistribution

Submit your manuscript at www.biomedcentral.com/submit
C Biomed Central 